\title{
Algebraic Structure of Adjoint Triples Generating a Weak Negation on the Unit Interval
}

\author{
M. Eugenia Cornejo ${ }^{(凶)}$, Jesús Medina, and Eloísa Ramírez-Poussa \\ Department of Mathematics, University of Cádiz, Cádiz, Spain \\ \{mariaeugenia.cornejo, jesus.medina, eloisa.ramirez\}@uca.es
}

\begin{abstract}
This paper presents a theoretical research about the relationship between weak negations and adjoint negations. Adjoint negations are a generalization of residuated negations built from the implications of an adjoint triple. Specifically, this work shows how to build adjoint triples on the unit interval such that their adjoint negations coincide with a given weak negation. Moreover, the algebraic structure formed by these adjoint triples is also investigated.
\end{abstract}

Keywords: Aggregation operator $\cdot$ Adjoint triple $\cdot$ Adjoint negation $\cdot$ Weak negation

\section{Introduction}

Non-monotonic operators play an important role in different applications $[7,9,29,31$, 38]. The need to use these operators in real applications has promoted the study and development of novel operators capable of addressing new challenges $[3,4,8,14,18,38$, $39]$. Weak negations were introduced in $[23,24,26,43]$ and they are one of the most versatile negation operators. For that reason, a generalization of weak negations was given in [14]. Specifically, it was proven that weak negations can be defined from the implications of adjoint triples.

Adjoint triples were introduced in [11-13] as a flexible tool to generalize the operators usually considered in residuated frameworks. One of the main advantages provided by these operators is the capability of being applied in non-associative or commutative settings. This fact has given rise to make more flexible frameworks such as logic programming [9,34-36], formal concept analysis [15,33], rough set theory [17], fuzzy relation equations $[10,20,21]$ and fuzzy mathematical morphology $[1,2,30]$.

In this paper, we will continue studying the relationship between weak negations and adjoint negations. Given a weak negation on the unit interval, we will show that different adjoint triples can be defined on the unit interval satisfying that its corresponding adjoint negations coincide with the weak negation. In addition, we will establish

\footnotetext{
Partially supported by the State Research Agency (AEI) and the European Regional Development Fund (ERDF) project TIN2016-76653-P, European Cooperation in Science \& Technology (COST) Action CA17124.
} 
two different procedures to define these adjoint triples. We will also define an ordering relation on adjoint triples generating a given weak negation and we will prove that the set composed of these adjoint triples has the structure of a complete join-semilattice with maximum element.

The paper is organized as follows: Sect. 2 recalls the basic definitions and properties associated with adjoint triples, adjoint negations and weak negations. Given a weak negation, in Sect. 3, we propose two different mechanisms to define adjoint triples on $[0,1]$ whose adjoint negations are equal to the considered weak negation. Furthermore, we analyze the algebraic structure formed by these adjoint triples. The contribution is accompanied by examples in order to illustrate some of the developed technical results. Some conclusions and prospects for future work are presented in Sect. 4.

\section{Preliminaries}

Adjoint triples provide an interesting generalization of the well-known adjoint property satisfied by a t-norm and its residuated implication, since they preserve the main properties usually assumed in residuated frameworks, dismissing for example the commutativity and the associativity [19].

Definition 1. Let $\left(P_{1}, \leq_{1}\right),\left(P_{2}, \leq_{2}\right),\left(P_{3}, \leq_{3}\right)$ be posets and $\&: P_{1} \times P_{2} \rightarrow P_{3}, \swarrow: P_{3} \times$ $P_{2} \rightarrow P_{1}, \nwarrow: P_{3} \times P_{1} \rightarrow P_{2}$ be mappings. We say that $(\&, \swarrow, \nwarrow)$ is an adjoint triple with respect to $P_{1}, P_{2}, P_{3}$ if the following double equivalence is satisfied:

$$
x \leq_{1} z \swarrow y \text { iff } x \& y \leq_{3} z \text { iff } y \leq_{2} z \nwarrow x
$$

for all $x \in P_{1}, y \in P_{2}$ and $z \in P_{3}$. The previous double equivalence is called adjoint property.

Interesting properties related to the monotonicity of the operators $\&, \swarrow, \nwarrow$, the boundary conditions and the preservation of the infimum and/or supremum, among others, can be deduced from the adjoint property. The following propositions show alternative ways to verify that the operators $\&, \swarrow$ and $\nwarrow$ form an adjoint triple, when they are defined on complete lattices.

Proposition 1 [13]. Given the complete lattices $\left(L_{1}, \unlhd_{1}\right),\left(L_{2}, \leq_{2}\right),\left(L_{3}, \preceq_{3}\right)$, an arbitrary operator \&: $L_{1} \times L_{2} \rightarrow L_{3}$ and the mappings $\swarrow: L_{3} \times L_{2} \rightarrow L_{1}, \nwarrow: L_{3} \times L_{1} \rightarrow L_{2}$, defined as $z \swarrow y=\sup \left\{x \in L_{1} \mid x \& y \leq_{3} z\right\}$ and $z \nwarrow x=\sup \left\{y \in L_{2} \mid x \& y \leq_{3}\right.$ $z\}$, respectively, for all $x \in L_{1}, y \in L_{2}$ and $z \in L_{3}$, the the following statements are equivalent:

1. (\&, $\swarrow, \nwarrow)$ is an adjoint triple with respect to $L_{1}, L_{2}, L_{3}$.

2. $\left(\bigvee_{x_{i} \in X} x_{i}\right) \& y=\bigvee_{x_{i} \in X}\left(x_{i} \& y\right)$, for any $X \subseteq L_{1}$ and $y \in L_{2}$.

$x \&\left(\bigvee_{y_{i} \in Y} y_{i}\right)=\bigvee_{y_{i} \in Y}\left(x \& y_{i}\right)$, for any $Y \subseteq L_{2}$ and $x \in L_{1}$. 
3. $z \swarrow y=\max \left\{x \in L_{1} \mid x \& y \leq_{3} z\right\}$ and $z \nwarrow x=\max \left\{y \in L_{2} \mid x \& y \leq_{3} z\right\}$ for all $x \in L_{1}, y \in L_{2}$ and $z \in L_{3}$, where \& is an order-preserving operator in both arguments.

Proposition 2 [13]. Given three complete lattices $\left(L_{1}, \leq_{1}\right),\left(L_{2}, \leq_{2}\right),\left(L_{3}, \leq_{3}\right)$, the arbitrary operators $\swarrow: L_{3} \times L_{2} \rightarrow L_{1}, \nwarrow: L_{3} \times L_{1} \rightarrow L_{2}$ and the mapping \& $L_{1} \times L_{2} \rightarrow L_{3}$ defined as $x \& y=\inf \left\{z \in L_{3} \mid x \leq_{1} z \swarrow y\right\}=\inf \left\{z \in L_{3} \mid y \leq_{2} z \nwarrow x\right\}$, for all $x \in L_{1}$ and $y \in L_{2}$, the following statements are equivalent:

1. (\&, $\swarrow, \nwarrow)$ is an adjoint triple with respect to $L_{1}, L_{2}, L_{3}$.

2. $\left(\bigwedge_{z_{i} \in Z} z_{i}\right) \swarrow y=\bigwedge_{z_{i} \in Z}\left(z_{i} \swarrow y\right)$, for all $Z \subseteq L_{3}$ and $y \in L_{2}$. $\left(\bigwedge_{z_{i} \in Z} z_{i}\right) \nwarrow x=\bigwedge_{z_{i} \in Z}\left(z_{i} \nwarrow x\right)$, for all $Z \subseteq L_{3}$ and $x \in L_{1}$.

3. $x \& y=\min \left\{z \in L_{3} \mid x \unlhd_{1} z \swarrow y\right\}=\min \left\{z \in L_{3} \mid y \unlhd_{2} z \nwarrow x\right\}$, for all $x \in L_{1}$ and $y \in L_{2}$, where $\swarrow$ and $\nwarrow$ are order-preserving operators in the first argument.

A detailed study of adjoint triples can be found in [11,13]. These operators were also considered to generalize residuated negations [6,25,40]. Specifically in [14], adjoint negations were defined from the implications of an adjoint triple. The formal definition of adjoint negations is given below.

Definition 2. Let $\left(P_{1}, \leq_{1}\right)$ and $\left(P_{2}, \leq_{2}\right)$ be two posets, $\left(P_{3}, \leq_{3}, \perp_{3}\right)$ be a lower bounded poset and $(\&, \swarrow, \nwarrow)$ an adjoint triple with respect to $P_{1}, P_{2}$ and $P_{3}$. The mappings $n_{n}: P_{1} \rightarrow P_{2}$ and $n_{s}: P_{2} \rightarrow P_{1}$ defined, for all $x \in P_{1}, y \in P_{2}$ as:

$$
n_{n}(x)=\perp_{3} \nwarrow x \quad n_{s}(y)=\perp_{3} \swarrow y
$$

are called adjoint negations with respect to $P_{1}$ and $P_{2}$. The operators $n_{s}$ and $n_{n}$ satisfying that $x=n_{s}\left(n_{n}(x)\right)$ and $y=n_{n}\left(n_{s}(y)\right)$, for all $x \in P_{1}$ and $y \in P_{2}$, are called strong adjoint negations.

Now, we will show the notion of weak negation which is one of the most general negation operators given in the literature [23,24,26,43].

Definition 3. Given a mapping $n:[0,1] \rightarrow[0,1]$ is said to be a weak negation if the following conditions hold, for all $x, y \in[0,1]$ :

1. $n(1)=0$;

2. if $x \leq y$ then $n(y) \leq n(x)$;

3. $x \leq n(n(x))$.

We say that $n$ is a strong negation if the equality $x=n(n(x))$ holds, for all $x \in[0,1]$.

Once the notion of weak negation has been introduced, we can recall the relationship between adjoint negations and weak negations. The following result, which was proven in [14], shows that adjoint negations are more general than weak negations. 
Theorem 1 [14]. If the mapping $n:[0,1] \rightarrow[0,1]$ is a weak negation, then there exists an adjoint triple $(\&, \swarrow, \nwarrow)$ with respect to the poset $([0,1], \leq)$ satisfying $n=n_{s}=n_{n}$.

Notice that, the previous theorem shows that weak negations can be obtained from the implication operators of an adjoint triple. However, we cannot guarantee the unicity of the adjoint triple which allows us to ensure that each weak negation is actually an adjoint negation. This fact and the notions introduced in the current section will be illustrated in the following example.

Example 1. The most usual adjoint triples with respect to $([0,1], \leq)$ are those defined from the Gödel, product and Łukasiewicz t-norms together with their residuated implications. Due to these t-norms are commutative, we have that $\swarrow^{\mathrm{G}}=\nwarrow_{\mathrm{G}}, \swarrow^{\mathrm{P}}=\nwarrow_{\mathrm{P}}$ and $\swarrow^{\mathrm{L}}=\nwarrow_{\mathrm{L}}$. As a consequence, the adjoint negations defined from these implications verify that $n_{s_{\mathrm{G}}}=n_{n_{\mathrm{G}}}, n_{s_{\mathrm{P}}}=n_{n_{\mathrm{P}}}$ and $n_{s_{\mathrm{L}}}=n_{s_{\mathrm{L}}}$. In order to simplify the notation, we will use $n_{\mathrm{G}}, n_{\mathrm{P}}$ and $n_{\mathrm{L}}$ to refer to the adjoint negations obtained from the Gödel, product and Łukasiewicz implications, respectively. The mentioned adjoint triples are given below:

$$
\begin{array}{rlrl}
\&_{\mathrm{G}}(x, y) & =\min \{x, y\} & z \swarrow^{\mathrm{G}} y & = \begin{cases}1 & \text { if } y \leq z \\
z & \text { otherwise }\end{cases} \\
\&_{\mathrm{P}}(x, y) & =x \cdot y & z \swarrow^{\mathrm{P}} y=\min \{1, z / y\} \\
\&_{\mathrm{L}}(x, y) & =\max \{0, x+y-1\} & z \swarrow^{\mathrm{L}} y=\min \{1,1-y+z\}
\end{array}
$$

Taking into account the of definition these operators, we obtain that the adjoint negations associated with the Gödel and product residuated implications are defined as:

$$
n_{\mathrm{G}}(x)=n_{\mathrm{P}}(x)= \begin{cases}1 & \text { if } x=0 \\ 0 & \text { otherwise }\end{cases}
$$

for all $x \in[0,1]$. From now on, this negation operator will be called product negation. In addition, the adjoint negation obtained from the Łukasiewicz residuated implication is defined as follows:

$$
n_{\mathrm{L}}(x)=1-x
$$

for all $x \in[0,1]$, and it is commonly known in the literature as the standard negation.

It is easy to see that the product negation is a weak negation whereas the standard negation is a strong negation. Obviously, we can ensure that the Gödel and product adjoint triples $\left(\&_{\mathrm{G}}, \swarrow^{\mathrm{G}}, \nwarrow_{\mathrm{G}}\right)$ and $\left(\&_{\mathrm{P}}, \swarrow^{\mathrm{P}}, \nwarrow_{\mathrm{P}}\right)$ verify Theorem 1 , for the weak negation $n_{\mathrm{P}}$. Hence, we can conclude that there exist at least two different adjoint triples whose adjoint negations coincide with the weak negation $n_{\mathrm{P}}$.

Next section studies how to define adjoint triples such that their adjoint negations are equal to a given weak negation. Furthermore, the algebraic structure formed by these adjoint triples is analyzed. 


\section{Adjoint Triples Generating a Weak Negation}

This section presents two different procedures to define adjoint triples whose adjoint negations coincide with a given weak negation. Besides, an ordering relation is defined on the whole set of adjoint triples generating a given weak negation. From this ordering relation, the algebraic structure composed of the aforementioned set of adjoint triples is obtained.

\subsection{Adjoint Triples Associated with Weak Negations from Adjoint Triples}

The first procedure to define adjoint triples generating a given weak negation $n$ is presented in the following proposition. This procedure is based on the use of an adjoint triple $(\&, \swarrow, \nwarrow)$ with respect to $([0,1], \leq)$ verifying the inequalities $0 \swarrow y \leq n(y)$ and $0 \nwarrow x \leq n(x)$, for all $x, y \in[0,1]$. From now on, given a weak negation $n$, the set of all adjoint triples with respect to $([0,1], \leq)$ such that their adjoint negations coincide with the weak negation $n$, we will denoted as $\mathcal{T}_{n}$.

Theorem 2. Let $n$ be a weak negation and $(\&, \swarrow, \nwarrow)$ be an adjoint triple with respect to $([0,1], \leq)$ such that $0 \swarrow y \leq n(y)$ and $0 \nwarrow x \leq n(x)$, for all $x, y \in[0,1]$. The

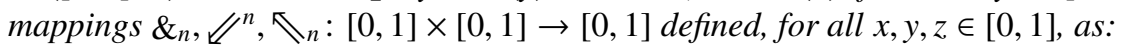

$$
\begin{gathered}
x \&_{n} y= \begin{cases}x \& y & \text { if } \quad x \not n(y) \\
0 & \text { if } \quad x \leq n(y)\end{cases} \\
z \mathscr{U}^{n} y=\max \{z \swarrow y, n(y)\} \quad z \nwarrow_{n} x=\max \{z \nwarrow x, n(x)\}
\end{gathered}
$$

form an adjoint triple with respect to $([0,1], \leq)$ belonging to $\mathcal{T}_{n}$, that is, they satisfy that $n=n_{s_{n}}=n_{n_{n}}$, where $n_{s_{n}}$ and $n_{n_{n}}$ are the adjoint negations defined from the implications $\mathscr{U}^{n}$ and $\nwarrow_{n}$, respectively.

Notice that, the previous result follows the idea presented in [5] for the construction of left continuous t-norms from a given weak negation. Specifically, Theorem 2 extends Lemma 1 introduced in [5] to the framework of adjoint triples.

Example 2. We will consider different adjoint triples with respect to $([0,1], \leq)$ generating the weak negation $n_{\mathrm{P}}$. We have considered this negation operator due to its simplicity and that it is not a strong negation. The first adjoint triple that we use in this example was already considered in previous works $[11,32]$. This adjoint triple $(\&, \swarrow, \nwarrow)$ with respect to $([0,1], \leq)$ is defined as follows:

$$
\begin{gathered}
x \& y=x^{2} \cdot y \\
z \swarrow y=\left\{\begin{array}{ll}
1 & \text { if } y=0 \\
\min \left\{\sqrt{\frac{z}{y}}, 1\right\} & \text { otherwise }
\end{array} \quad z \nwarrow x= \begin{cases}1 & \text { if } x=0 \\
\min \left\{\frac{z}{x^{2}}, 1\right\} & \text { otherwise }\end{cases} \right.
\end{gathered}
$$


for all $x, y, z \in[0,1]$. It is easy to check that the following inequalities $0 \swarrow y \leq n_{\mathrm{P}}(y)$ and $0 \nwarrow x \leq n_{\mathrm{P}}(x)$ are satisfied, for all $x, y \in[0,1]$. Indeed, the inequalities are equalities in this case. Hence, by using Theorem 2, we can define the following operators:

$$
\begin{aligned}
& x \&_{n_{\mathrm{P}}}^{1} y=\left\{\begin{array}{ll}
x \& y & \text { if } x \not n_{\mathrm{P}}(y) \\
0 & \text { if } x \leq n_{\mathrm{P}}(y)
\end{array}=\left\{\begin{array}{ll}
x^{2} \cdot y & \text { if } x \not \leq n_{\mathrm{P}}(y) \\
0 & \text { if } x \leq n_{\mathrm{P}}(y)
\end{array}=x^{2} \cdot y\right.\right. \\
& z \mathscr{U}^{n_{P 1}} y=\max \left\{z \swarrow y, n_{\mathrm{P}}(y)\right\}= \begin{cases}1 & \text { if } y=0 \\
\min \left\{\sqrt{\frac{z}{y}}, 1\right\} & \text { otherwise }\end{cases} \\
& z \nwarrow_{n_{P 1}} x=\max \left\{z \nwarrow x, n_{\mathrm{P}}(x)\right\}= \begin{cases}1 & \text { if } x=0 \\
\min \left\{\frac{z}{x^{2}}, 1\right\} & \text { otherwise }\end{cases}
\end{aligned}
$$

Clearly, the adjoint triple $\left(\&_{n_{\mathrm{P}}}^{1}, \mathscr{l}^{n_{P 1}}, \mathbb{}_{n_{P 1}}\right)$ defined as in Theorem 2 coincides with $(\&, \swarrow, \nwarrow)$. Notice that, the adjoint triple $\left(\&_{n_{\mathrm{P}}}^{1}, \mathscr{U}^{n_{P 1}}, \mathbb{}_{n_{P 1}}\right)$ belongs to $\mathcal{T}_{n_{\mathrm{P}}}$ since the following chains of equalities hold, for all $x, y \in[0,1]$ :

$$
\begin{aligned}
& n_{s_{n_{P 1}}}(y)=0 \mathscr{U}^{n_{P 1}} y=\left\{\begin{array}{ll}
1 & \text { if } y=0 \\
0 & \text { otherwise }
\end{array}=n_{\mathrm{P}}(y)\right. \\
& n_{n_{n_{P 1}}}(x)=0 \mathbb{n}_{n_{P 1}} x=\left\{\begin{array}{ll}
1 & \text { if } x=0 \\
0 & \text { otherwise }
\end{array}=n_{\mathrm{P}}(x)\right.
\end{aligned}
$$

The implications associated with the Gödel adjoint triple $\left(\&_{\mathrm{G}}, \swarrow^{\mathrm{G}}, \nwarrow_{\mathrm{G}}\right)$ also verify the hypothesis required in Theorem 2, that is, the inequalities $0 \swarrow^{\mathrm{G}} y=n_{\mathrm{G}}(y) \leq n_{\mathrm{P}}(y)$ and $0 \nwarrow_{\mathrm{G}} x=n_{\mathrm{G}}(x) \leq n_{\mathrm{P}}(x)$ trivially hold, for all $x, y \in[0,1]$. Consequently, applying Theorem 2, we can define the following operators:

$$
\begin{gathered}
x \&_{n_{\mathrm{P}}}^{2} y=\left\{\begin{array}{ll}
x \&_{\mathrm{G}} y & \text { if } x \not n_{\mathrm{P}}(y) \\
0 & \text { if } x \leq n_{\mathrm{P}}(y)
\end{array}=\left\{\begin{array}{ll}
\min \{x, y\} & \text { if } x \leq n_{\mathrm{P}}(y) \\
0 & \text { if } x \leq n_{\mathrm{P}}(y)
\end{array}=\min \{x, y\}\right.\right. \\
z \mathscr{U}^{n_{P 2}} y=\max \left\{z \swarrow^{\mathrm{G}} y, n_{\mathrm{P}}(y)\right\}= \begin{cases}1 & \text { if } y \leq z \\
z & \text { otherwise }\end{cases}
\end{gathered}
$$

Therefore, we also have that $\left(\&_{n_{\mathrm{P}}}^{2}, \bigcup^{n_{P 2}}, \mathbb{}_{n_{P 2}}\right)$ defined as in Theorem 2 coincides with $\left(\&_{\mathrm{G}}, \swarrow^{\mathrm{G}}, \nwarrow_{\mathrm{G}}\right)$. Indeed, this fact will arise to every adjoint triple satisfying the hypotheses in Theorem 2, due to the restrictive definition of the negation operator $n_{\mathrm{P}}$. As previously, the following chain of equalities is satisfied, for all $y \in[0,1]$ :

$$
n_{s_{n_{P 2}}}(y)=0 \mathscr{U}^{n_{P 2}} y=n_{\mathrm{P}}(y)=0 \mathbb{N}_{n_{P 2}} y=n_{n_{n_{P 2}}}(y)
$$

Thus, $\left(\&_{n_{\mathrm{P}}}^{2}, \mathscr{U}^{n_{P 2}}, \mathbb{}_{n_{P 2}}\right) \in \mathcal{T}_{n_{\mathrm{P}}}$, that is, it is an adjoint triple generating the weak negation $n_{\mathrm{P}}$. Obviously, the product adjoint triple $\left(\&_{\mathrm{P}}, \swarrow^{\mathrm{P}}, \nwarrow_{\mathrm{P}}\right)$ also belongs to $\mathcal{T}_{n_{\mathrm{P}}}$. Notice that, the Łukasiewicz adjoint triple $\left(\&_{\mathrm{L}}, \swarrow^{\mathrm{L}}, \nwarrow_{\mathrm{L}}\right)$ cannot be considered to build an adjoint triple whose adjoint negations coincide with the weak negation $n_{\mathrm{P}}$. This fact is due to that the hypothesis required in Theorem 2 are not satisfied. For example, if we consider $y=0.2$ and $z=0$, we have that:

$$
0 \swarrow^{\mathrm{L}} 0.2=\min \{1,1-0.2+0\}=0.8 \not \leq 0=n_{P}(0.2)
$$




\subsection{Adjoint Triples Associated with Weak Negations from Sup-Homomorphisms}

The following result establishes the second procedure to define adjoint triples generating a given weak negation. This second mechanism weakens the required conditions in the first procedure and considers more general operators than adjoint triples. In particular, the proposed mechanism is based on the use of mappings preserving the supremum of non-empty sets, which are called supremum-homomorphisms on lattice theory.

Theorem 3. Let $n$ be a weak negation, $f, g, h:[0,1] \times[0,1] \rightarrow[0,1]$ three mappings such that $f$ preserves the supremum of non-empty sets in both arguments, $g$ is defined as $g(z, y)=\sup \{x \in[0,1] \mid f(x, y) \leq z\}$ satisfying that $g(0, y) \leq n(y)$ and $h$ is defined as $h(z, x)=\sup \{y \in[0,1] \mid f(x, y) \leq z\}$ satisfying that $h(0, x) \leq n(x)$, for all $x, y, z \in[0,1]$. The triple $\left(\&_{n}, \mathscr{U}^{n}, \mathbb{\bigotimes}_{n}\right)$ composed of the following operators:

$$
\begin{gathered}
x \&_{n} y= \begin{cases}f(x, y) & \text { if } \quad x \not n(y) \\
0 & \text { if } \quad x \leq n(y)\end{cases} \\
z \mathscr{U}^{n} y=\max \{g(z, y), n(y)\} \quad z \mathbb{N}_{n} \quad x=\max \{h(z, x), n(x)\}
\end{gathered}
$$

is an adjoint triple with respect to $([0,1], \leq)$ of $\mathcal{T}_{n}$.

As a consequence of this result, general operators can be considered to define adjoint triples in $\mathcal{T}_{n}$, such as uninorms $[13,22,27,41,42,44]$. This fact notably increases the number of operators that can be considered for obtaining triples in $\mathcal{T}_{n}$, which has a direct consequence in the flexibility for using these operators in real cases.

Example 3. In this example we will consider the uninorm $f:[0,1] \times[0,1] \rightarrow[0,1]$, defined for all $x, y \in[0,1]$ as follows.

$$
f(x, y)= \begin{cases}\min \{x, y\} & \text { if } x \leq \frac{1}{2} \text { and } y \leq \frac{1}{2} \\ \max \{x, y\} & \text { otherwise }\end{cases}
$$

It is easy to check that $f$ preserves the supremum of non-empty sets in both arguments. Moreover, from $f$, we can define two mappings $g, h:[0,1] \times[0,1] \rightarrow[0,1]$ as $g(z, y)=\sup \{x \in[0,1] \mid f(x, y) \leq z\}$ and $h(z, x)=\sup \{y \in[0,1] \mid f(x, y) \leq z\}$, for all $x, y, z \in[0,1]$. Notice that, $f$ is a commutative mapping and therefore $g=h$. The analytic expression of the mapping $g$ is displayed below:

$$
g(z, y)=\sup \{x \in[0,1] \mid f(x, y) \leq z\}= \begin{cases}\frac{1}{2} & \text { if } y \leq z \leq \frac{1}{2} \\ z & \text { if } y \leq z \text { and } z>\frac{1}{2} \\ 0 & \text { otherwise }\end{cases}
$$

Clearly, the inequality $g(0, y) \leq n_{\mathrm{P}}(y)$ holds for all $y \in[0,1]$, and consequently $h(0, x) \leq n_{\mathrm{P}}(x)$, for all $x \in[0,1]$. Under the hypothesis of Theorem 3 , we can define 
an adjoint triple $\left(\&_{n_{\mathrm{P}}}^{3}, \mathscr{U}^{n_{P 3}}, \mathbb{N}_{n_{P 3}}\right)$ from the mappings $f, g$ and $h$ such that it belongs to $\mathcal{T}_{n_{\mathrm{P}}}$. Specifically, the analytical expression of the conjunctor $\&_{n_{\mathrm{P}}}^{3}$ is:

$$
x \&_{n_{\mathrm{P}}}^{3} y=\left\{\begin{array}{lll}
f(x, y) & \text { if } & x \not n_{\mathrm{P}}(y) \\
0 & \text { if } & x \leq n_{\mathrm{P}}(y)
\end{array}= \begin{cases}0 & \text { if } x=0 \text { or } y=0 \\
\min \{x, y\} & \text { if } x, y \in\left(0, \frac{1}{2}\right] \\
\max \{x, y\} & \text { otherwise }\end{cases}\right.
$$

In this case, $\&_{n_{\mathrm{P}}}^{3}$ does not coincided with $f$. As the conjunctor $\&_{n_{\mathrm{P}}}^{3}$ is commutative, we have that $\mathscr{U}^{n_{P 3}}=\mathbb{}_{n_{P 3}}$. For all $y, z \in[0,1]$, the implication $\mathscr{U}^{n_{P 3}}$ is defined as:

$$
z \mathscr{U}^{n_{P 3}} y=\max \left\{g(z, y), n_{\mathrm{P}}(y)\right\}= \begin{cases}1 & \text { if } y=0 \\ z & \text { if } 0<y \leq z \text { and } z>\frac{1}{2} \\ \frac{1}{2} & \text { if } 0<y \leq z \leq \frac{1}{2} \\ 0 & \text { otherwise }\end{cases}
$$

As we mentioned above, $\left(\&_{n_{\mathrm{P}}}^{3}, \mathscr{U}^{n_{P 3}}, \mathbb{N}_{n_{P 3}}\right) \in \mathcal{T}_{n_{\mathrm{P}}}$ since the following chain of equalities is verified, for all $y \in[0,1]$ :

$$
n_{s_{n_{P 3}}}(y)=0 \mathscr{U}^{n_{P 3}} y=n_{\mathrm{P}}(y)=0 \mathbb{n}_{n_{P 3}} y=n_{n_{n_{P 3}}}(y)
$$

It is important to emphasize that $f$ does not preserve the supremum of non-empty sets in both arguments. For instance, when $X=\varnothing$ and $y=1$, we have that:

$$
f\left(\bigvee_{x_{i} \in X} x_{i}, y\right)=f(0,1)=1 \neq 0=\bigvee_{x_{i} \in X} f\left(x_{i}, 1\right)=\bigvee_{x_{i} \in X} f\left(x_{i}, y\right)
$$

As a consequence, $f$ cannot be the conjunctor of an adjoint triple. This fact allows us to ensure that the mechanism given in Theorem 3 provides adjoint triples built from more general operators.

\subsection{Algebraic Structure of $\mathcal{T}_{n}$}

The following theorem includes a point-wise ordering relation defined on the conjunctors of adjoint triples generating a given weak negation. This ordering relation provides the set of adjoint triples, whose adjoint negations coincide with such a weak negation, with the structure of a complete join-semilattice.

Theorem 4. Given a weak negation $n$, we have that the pair $\left(\mathcal{T}_{n}\right.$, $)$ forms a complete join-semilattice, where $\sqsubseteq$ is the ordering relation defined as:

$$
\left(\&_{n}^{j}, \mathscr{U}^{n_{j}}, \mathbb{N}_{n_{j}}\right) \sqsubseteq\left(\&_{n}^{k}, \mathscr{U}^{n_{k}}, \mathbb{\}_{n_{k}}\right) \quad \text { iff } \quad x \&_{n}^{j} y \leq x \&_{n}^{k} y
$$


for all $x, y \in[0,1]$ and $\left(\&_{n}^{j}, \mathscr{U}^{n_{j}}, \mathbb{}_{n_{j}}\right),\left(\&_{n}^{k}, \mathscr{U}^{n_{k}}, \mathbb{}_{n_{k}}\right) \in \mathcal{T}_{n}$. The greatest element in $\mathcal{T}_{n}$ is the adjoint triple $\left(\&_{n}^{g}, \mathscr{U}^{n_{g}}, \mathbb{}_{n_{g}}\right)$ such that $\mathscr{U}^{n_{g}}=\mathbb{N}_{n_{g}}$, which is defined as follows:

$$
x \&_{n}^{g} y=\left\{\begin{array}{lll}
1 & \text { if } & x \not \leq n(y) \\
0 & \text { if } & x \leq n(y)
\end{array} \quad z \mathscr{U}^{n_{g}} \quad y= \begin{cases}n(y) & \text { if } \quad z \neq 1 \\
1 & \text { if } \quad z=1\end{cases}\right.
$$

for all $x, y, z \in[0,1]$.

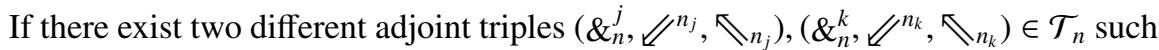
that $\left(\&_{n}^{j}, \mathscr{U}^{n_{j}}, \mathbb{N}_{n_{j}}\right) \nsubseteq\left(\&_{n}^{k}, \mathscr{U}^{n_{k}}, \mathbb{N}_{n_{k}}\right)$ and $\left(\&_{n}^{k}, \mathscr{U}^{n_{k}}, \mathbb{}_{n_{k}}\right) \nsubseteq\left(\&_{n}^{j}, \mathscr{U}^{n_{j}}, \mathbb{N}_{n_{j}}\right)$, then we will say that these adjoint triples are incomparable. In this case, we will write that $\left(\&_{n}^{j}, \mathscr{U}^{n_{j}}, \mathbb{N}_{n_{j}}\right) \|\left(\&_{n}^{k}, \mathscr{U}^{n_{k}}, \mathbb{N}_{n_{k}}\right)$.

Finally, we clarify the previous result by means of the following example. This example will be used to illustrate that the set $\left(\mathcal{T}_{n}, \sqsubseteq\right)$ has not the structure of a complete lattice, since the infimum of the elements in $\mathcal{T}_{n}$ could not necessarily belong to $\mathcal{T}_{n}$.

Example 4. Given the weak negation $n_{\mathrm{P}}$, we will establish a hierarchy among the proposed adjoint triples in $\mathcal{T}_{n_{\mathrm{P}}}$. According to the ordering relation introduced in Theorem 4, we obtain that:

$$
\left(\&_{n_{\mathrm{P}}}^{1}, \mathscr{l}^{n_{P 1}}, \mathbb{}_{n_{P 1}}\right) \sqsubseteq\left(\&_{\mathrm{P}}, \swarrow^{\mathrm{P}}, \nwarrow_{\mathrm{P}}\right) \sqsubseteq\left(\&_{n_{\mathrm{P}}}^{2}, \mathscr{U}^{n_{P 2}}, \mathbb{}_{n_{P 2}}\right) \sqsubseteq\left(\&_{n_{\mathrm{P}}}^{3}, \mathscr{U}^{n_{P 3}}, \mathbb{}_{n_{P 3}}\right)
$$

Although we can find other adjoint triples belonging to $\mathcal{T}_{n_{\mathrm{p}}}$ greater than the previous ones, by Theorem 4 , we can ensure that the greatest adjoint triple in $\mathcal{T}_{n_{\mathrm{P}}}$ is the triple $\left(\&_{g}, \swarrow^{g}, \nwarrow_{g}\right)$ such that $\swarrow^{g}=\nwarrow_{g}$, which is defined, for all $x, y, z \in[0,1]$, as follows:

$$
x \&_{g} y=\left\{\begin{array}{lll}
1 & \text { if } & x \not \leq n_{\mathrm{P}}(y) \\
0 & \text { if } \quad x \leq n_{\mathrm{P}}(y)
\end{array} \quad z \swarrow^{g} y= \begin{cases}n_{\mathrm{P}}(y) & \text { if } \quad z \neq 1 \\
1 & \text { if } \quad z=1\end{cases}\right.
$$

Finally, we will show that there exist incomparable adjoint triples in $\mathcal{T}_{n_{\mathrm{P}}}$ and, therefore, the complete join-semilattice $\left(\mathcal{T}_{n_{\mathrm{P}}}, \sqsubseteq\right)$ is not linear. Given $a \in(0,1)$, we can define the operators $\&_{a}, \swarrow^{a}, \nwarrow_{a}$ on the unit interval such that $\swarrow^{a}=\nwarrow_{a}$ as follows:

$$
x \&_{a} y=\left\{\begin{array}{lll}
a & \text { if } & x \not n_{\mathrm{P}}(y) \\
0 & \text { if } & x \leq n_{\mathrm{P}}(y)
\end{array} \quad z \swarrow^{a} y=\left\{\begin{array}{lll}
n_{\mathrm{P}}(y) & \text { if } & a \not z z \\
1 & \text { if } & a \leq z
\end{array}\right.\right.
$$

In particular, these triples are incomparable with the adjoint triples previously defined $\left(\&_{\mathrm{P}}, \swarrow^{\mathrm{P}}, \nwarrow_{\mathrm{P}}\right),\left(\&_{n_{\mathrm{P}}}^{2}, \swarrow^{n_{P 2}}, \mathbb{}_{n_{P 2}}\right)$ and $\left(\&_{n_{\mathrm{P}}}^{3}, \mathscr{U}^{n_{P 3}}, \mathbb{}_{n_{P 3}}\right)$. Considering $a=0.45$, then the adjoint triple $\left(\&_{0.45}, \swarrow^{0.45}, \nwarrow_{0.45}\right)$ verifies that:

$$
\left.\begin{array}{rl}
0.8 \&_{0.45} 0.6=0.45 & \leq 0.48=0.8 \&_{\mathrm{P}} 0.6 \\
0.5 \&_{\mathrm{P}} 0.6=0.3 & \leq 0.45=0.5 \&_{0.45} 0.6
\end{array}\right\} \text { then }\left(\&_{\mathrm{P}}, \swarrow^{\mathrm{P}}, \nwarrow_{\mathrm{P}}\right) \|\left(\&_{0.45}, \swarrow^{0.45}, \nwarrow_{0.45}\right)
$$


Thus, when the weak negation $n_{\mathrm{P}}$ is considered, a proper non linear complete joinsemilattice arises. Moreover, $\left(\mathcal{T}_{n_{\mathrm{P}}}, \sqsubseteq\right)$ is not a complete lattice since the infimum of the subset $\left\{\left(\&_{a}, \swarrow^{a}, \nwarrow_{a}\right) \mid a \in(0,1]\right\} \subseteq \mathcal{T}_{n_{\mathrm{P}}}$ is the adjoint triple whose conjunctor is constantly zero, which is not an adjoint conjunctor of an adjoint triple in $\mathcal{T}_{n_{\mathrm{p}}}$.

\section{Conclusions and Future Work}

We have extended the studied carried out in [14], providing different procedures to determine adjoint triples on the unit interval, whose adjoint negations are actually a previously fixed weak negation. We have also defined an ordering on which the set of these adjoint triples forms a complete join-semilattice. In addition, we have characterized the maximum element of the mentioned complete join-semilattice. In order to clarify the developed theory in this paper, we have included some illustrative examples.

As a future work, we will apply the developed theoretical results to different frameworks such as formal concept analysis, fuzzy relation equations and rough set theory. For example, these results will be fundamental for studying families of adjoint triples for defining preferences on objects or/and attributes in relational datasets [16,28,37]. This fact will allow us to address real problems related to image processing and digital forensic analysis.

Acknowledgement. Partially supported by the 2014-2020 ERDF Operational Programme in collaboration with the State Research Agency (AEI) in projects TIN2016-76653-P and PID2019108991GB-I00, and with the Department of Economy, Knowledge, Business and University of the Regional Government of Andalusia in project FEDER-UCA18-108612, and by the European Cooperation in Science \& Technology (COST) Action CA17124.

\section{References}

1. Alcalde, C., Burusco, A., Díaz-Moreno, J., Fuentes-González, R., Medina, J.: Fuzzy property-oriented concept lattices in morphological image and signal processing. In: Rojas, I., Joya, G., Cabestany, J. (eds.) IWANN 2013. LNCS, pp. 246-253. Springer, Heidelberg (2013). https://doi.org/10.1007/978-3-642-38682-4_28

2. Alcalde, C., Burusco, A., Díaz-Moreno, J.C., Medina, J.: Fuzzy concept lattices and fuzzy relation equations in the retrieval processing of images and signals. Int. J. Uncertain. Fuzziness Knowl.-Based Syst. 25(Supplement-1), 99-120 (2017)

3. Asiain, M.J., Bustince, H., Mesiar, R., Kolesárová, A., Takáč, Z.: Negations with respect to admissible orders in the interval-valued fuzzy set theory. IEEE Trans. Fuzzy Syst. 26(2), 556-568 (2018)

4. Chajda, I.: A representation of residuated lattices satisfying the double negation law. Soft. Comput. 22(6), 1773-1776 (2017)

5. Cignoli, R., Esteva, F., Godo, L., Montagna, F.: On a class of left-continuous t-norms. Fuzzy Sets Syst. 131(3), 283-296 (2002)

6. Cintula, P., Klement, E.P., Mesiar, R., Navara, M.: Residuated logics based on strict triangular norms with an involutive negation. Math. Log. Q. 52(3), 269-282 (2006)

7. Cintula, P., Klement, E.P., Mesiar, R., Navara, M.: Fuzzy logics with an additional involutive negation. Fuzzy Sets Syst. 161(3), 390-411 (2010). Fuzzy Logics and Related Structures 
8. Cornejo, M.E., Esteva, F., Medina, J., Ramírez-Poussa, E.: Relating adjoint negations with strong adjoint negations. In: Kóczy, J.M.L. (ed.) Proceedings 7th European Symposium on Computational Intelligence and Mathematics, pp. 66-71 (2015)

9. Cornejo, M.E., Lobo, D., Medina, J.: Syntax and semantics of multi-adjoint normal logic programming. Fuzzy Sets Syst. 345, 41-62 (2018)

10. Cornejo, M.E., Lobo, D., Medina, J.: On the solvability of bipolar max-product fuzzy relation equations with the product negation. J. Comput. Appl. Math. 354, 520-532 (2019)

11. Cornejo, M.E., Medina, J., Ramírez-Poussa, E.: A comparative study of adjoint triples. Fuzzy Sets Syst. 211, 1-14 (2013)

12. Cornejo, M.E., Medina, J., Ramírez-Poussa, E.: Multi-adjoint algebras versus extended-order algebras. Appl. Math. Inf. Sci. 9(2L), 365-372 (2015)

13. Cornejo, M.E., Medina, J., Ramírez-Poussa, E.: Multi-adjoint algebras versus noncommutative residuated structures. Int. J. Approx. Reason. 66, 119-138 (2015)

14. Cornejo, M.E., Medina, J., Ramírez-Poussa, E.: Adjoint negations, more than residuated negations. Inf. Sci. 345, 355-371 (2016)

15. Cornejo, M.E., Medina, J., Ramírez-Poussa, E.: Characterizing reducts in multi-adjoint concept lattices. Inf. Sci. 422, 364-376 (2018)

16. Cornejo, M.E., Medina, J., Ramírez-Poussa, E., Rubio-Manzano, C.: Multi-adjoint concept lattices, preferences and Bousi Prolog. In: Flores, V., et al. (eds.) IJCRS 2016. LNCS, vol. 9920, pp. 331-341. Springer, Cham (2016). https://doi.org/10.1007/978-3-319-47160-0_30

17. Cornelis, C., Medina, J., Verbiest, N.: Multi-adjoint fuzzy rough sets: Definition, properties and attribute selection. Int. J. Approx. Reason. 55, 412-426 (2014)

18. Della Stella, M.E., Guido, C.: Associativity, commutativity and symmetry in residuated structures. Order 30(2), 363-401 (2013)

19. Demirli, K., De Baets, B.: Basic properties of implicators in a residual framework. Tatra Mount. Math. Publ. 16, 31-46 (1999)

20. Díaz-Moreno, J.C., Medina, J.: Multi-adjoint relation equations: definition, properties and solutions using concept lattices. Inf. Sci. 253, 100-109 (2013)

21. Díaz-Moreno, J.C., Medina, J.: Using concept lattice theory to obtain the set of solutions of multi-adjoint relation equations. Inf. Sci. 266, 218-225 (2014)

22. Duan, Q., Zhao, B.: Maximal chains on the interval $[0,1]$ with respect to t-norm-partial orders and uninorm-partial orders. Inf. Sci. 516, 419-428 (2020)

23. Esteva, F.: Negaciones en retículos completos. Stochastica I, 49-66 (1975)

24. Esteva, F., Domingo, X.: Sobre funciones de negación en [0,1]. Stochastica IV, 141-166 (1980)

25. Esteva, F., Godo, L., Hájek, P., Navara, M.: Residuated fuzzy logics with an involutive negation. Arch. Math. Log. 39(2), 103-124 (2000)

26. Esteva, F., Trillas, E., Domingo, X.: Weak and strong negation functions in fuzzy set theory. In: Proceedings of the XI International Symposium on Multivalued Logic, pp. 23-26 (1981)

27. Jenei, S.: Introducing group-like uninorms-construction and characterization. In: Kóczy, L., Medina-Moreno, J., Ramírez-Poussa, E., Šostak, A. (eds.) Computational Intelligence and Mathematics for Tackling Complex Problems. Studies in Computational Intelligence, vol. 819, pp. 51-57. Springer, Cham (2020). https://doi.org/10.1007/978-3-030-16024-1_7

28. Khan, M.A.: Formal reasoning in preference-based multiple-source rough set model. Inf. Sci. 334-335, 122-143 (2016)

29. Madrid, N., Ojeda-Aciego, M.: Measuring inconsistency in fuzzy answer set semantics. IEEE Trans. Fuzzy Syst. 19(4), 605-622 (2011)

30. Madrid, N., Ojeda-Aciego, M., Medina, J., Perfilieva, I.: L-fuzzy relational mathematical morphology based on adjoint triples. Inf. Sci. 474, 75-89 (2019)

31. Massanet, S., Recasens, J., Torrens, J.: Fuzzy implication functions based on powers of continuous t-norms. Int. J. Approx. Reason. 83, 265-279 (2017) 
32. Medina, J., Ojeda-Aciego, M.: Multi-adjoint t-concept lattices. Inf. Sci. 180(5), 712-725 (2010)

33. Medina, J., Ojeda-Aciego, M., Ruiz-Calviño, J.: Formal concept analysis via multi-adjoint concept lattices. Fuzzy Sets Syst. 160(2), 130-144 (2009)

34. Medina, J., Ojeda-Aciego, M., Vojtáš, P.: Multi-adjoint logic programming with continuous semantics. In: Eiter, T., Faber, W., Truszczyński, M. (eds.) LPNMR 2001. LNCS, vol. 2173, pp. 351-364. Springer, Berlin, Heidelberg (2001). https://doi.org/10.1007/3-54045402-0_26

35. Medina, J., Ojeda-Aciego, M., Vojtáš, P.: Similarity-based unification: a multi-adjoint approach. Fuzzy Sets Syst. 146, 43-62 (2004)

36. Moreno, G., Penabad, J., Vázquez, C.: Beyond multi-adjoint logic programming. Int. J. Comput. Math. 92(9), 1956-1975 (2015)

37. Pan, W., She, K., Wei, P.: Multi-granulation fuzzy preference relation rough set for ordinal decision system. Fuzzy Sets Syst. 312, 87-108 (2017). Theme: Fuzzy Rough Sets

38. Pradera, A., Beliakov, G., Bustince, H., Baets, B.D.: A review of the relationships between implication, negation and aggregation functions from the point of view of material implication. Inf. Sci. 329, 357-380 (2016)

39. Pradera, A., Massanet, S., Ruiz-Aguilera, D., Torrens, J.: The non-contradiction principle related to natural negations of fuzzy implication functions. Fuzzy Sets Syst. 359, 3-21 (2019)

40. San-Min, W.: Logics for residuated pseudo-uninorms and their residua. Fuzzy Sets Syst. 218, 24-31 (2013)

41. Su, Y., Liu, H.-W., Pedrycz, W.: The distributivity equations of semi-uninorms. Int. J. Uncertain. Fuzziness Knowl.-Based Syst. 27(02), 329-349 (2019)

42. Su, Y., Riera, J., Ruiz-Aguilera, D., Torrens, J.: The modularity condition for uninorms revisited. Fuzzy Sets Syst. 357, 27-46 (2019). Theme: Aggregation Functions

43. Trillas, E.: Sobre negaciones en la teoría de conjuntos difusos. Stochastica III, 47-60 (1979)

44. Zong, W., Su, Y., Liu, H.-W., Baets, B.D.: On the construction of uninorms by paving. Int. J. Approx. Reason. 118, 96-111 (2020) 